\title{
RESPONSES
}

\section{Sexe, mensonge et vidéo ${ }^{1}$}

Sylvie Frigon

In this commentary, the author exposes the (en)gendered violence done to incarcerated women at Canada's Prison For Women (P4W) during the strip search of April 26-27, 1994 conducted by the Institutional Emergency Response Team (IERT) and raises issues concerning the segregation Post-April, 1994. The legitimacy of this intervention, the "degradation ceremonies" in operation and the production of "docile bodies" are raised and critiqued in view of the situation of imprisoned women in Canada.

O commentaire vise à exposer et dénoncer la violence faite aux femmes détenues dans le système carcéral canadien, en prenant comme pivot d'analyse un segment du vidéo sur l'intervention de l'escouade anti-émeute au pénitencier pour femmes à Kingston - mieux connu sous l'acronyme P4W (prison for women) ${ }^{2}$ - dans lequel on exhibe des femmes mises à nu, enchaînées et mâtées au nom de la sécurité.

Cette discussion sera découpée en 5 actes: (1) de la théâtralité de la justice; (2) intervention policière ou "rituels de dégradation"?; (3) le corps mâté, le corps "docile"; (4) sur l'enfermement carcéral des femmes; et (5) de l'indignation au silence.

\section{De la théatralité de la justice}

La place de la télédiffusion dans le domaine de la justice est d'actualité en Amérique du Nord. De la Cour en direct au procès d'O.J. Simpson, on joue sur la théâtralité de la justice. Au Canada, on en redemande. On veut nos propres procès télévisés - Made in Canada sous le prétexte que l'on connaît mieux le système de justice américain. La fascination pour le procès d'O.J. Simpson en est un exemple intéressant. C'est L.A. Law, mais en vrai. C'est me semble-t-il, la continuation des émissions comme celle d'enquête criminelle 24 heures sur 24. On ne se contente plus de la fiction, on réclame la "vraie" vie. D'ailleurs, il n'est pas surprenant d'assister à la prolifération d'émissions comme Claire Lamarche, Oprah, Shirley, pour ne nommer que celles-ci. 
Certains diront que l'on donne la parole et la voix aux gens "ordinaires" et d'autres accuseront ces émissions de voyeurisme et de sensationnalisme. Au nom de la justice, le voyeurisme est légitimé. Mais la justice est bien loin de nos préoccupations.

Les affaires O'Carroll au Québec et Rodney King aux Etats-Unis, les émeutes de Los Angeles, les rites d'initiation du Régiment aéroporté de Petawawa en Ontario, tous ont une chose en commun: des images sur bande vidéo qui influencent et même changent le déroulement des événements. Des images qui permettent de dénoncer la violence policière et porter formellement des accusations contre des policiers violents, parois de rendre justice et d'autres fois, de faire démanteler des régiments. Des vidéos amateurs et/ou clandestins. A la différence de ces derniers, celui de l'intervention de l'escouade anti-émeute au pénitencier pour femmes au Canada en est un tourné par un membre de cette escouade. Ainsi, il s'agit d'un vidéo "institutionnel" qui, selon The Globe and Mail du 21 février 1995, a été obtenu par la CBC, par l'avocat d'une des détenues ${ }^{3}$. Ceci nous amène à nous demander, par exemple, si nous croirions uniquement les récits d'abus des orphelins de Duplessis, les abus sexuels des enfants, la violence conjugale s'ils étaient enregistrés sur bande vidéo?... Pour avoir justice, faudra-t-il demander à Monsieur Béliveau de Surprise sur prise d'enregistrer sur bobine, nos horreurs? Pour notre plaisir les dimanches soirs...

\section{Intervention policière ou "rituels de dégradation"?}

Dans un article intitulé "Les insolences d'un caméscope" de l'édition des 25 et 26 février demier du quotidien Le Devoir, Pierre Cayouette se demande si 'le coté pervers de l'affaire, c'est que ces vidéos risquent de court-circuiter le réflexe critique du téléspectateur. Le public saute tout de suite aux conclusions ... ." Il demande, par exemple, "[q]ui a retenu, en regardant les images de la prison de Kingston, que cette brutale et très contestable intervention policière faisait suite à quatre jours de troubles graves ...?" (je souligne). C'est un argument qui est au mieux, mince et au pire, peut etre dangereux puisque selon ce que l'on peut voir dans le document visuel présenté à The 5th Estate le 21 février, lorsque les agents, très majoritairement masculins ${ }^{4}$ font irruption dans les cellules des détenues, tout était calme: certaines dormaient et la plupart n'offraient aucune résistance. Alors, au moment précis de l'intervention, 
elles ne représentaient pas de danger, n'est-ce pas? Alors où est la justification pour ces actes de répression? Et, ces rituels de dégradation qui ont eu comme conséquence la ségrégation des femmes impliquées dans les troubles d'avril 1995 pendant huit à neuf mois, c'cst-à-dire enfermées 23 heures sur 24 ! Et, cette violation des droits de la personne?

De plus, les règlements des institutions d'incarcération stipulent que - sauf en situation d'urgence - des fouilles à nu sur des détenues ne peuvent être faites que par des agents de sexe féminin. Même la Cour suprême du Canada a déjà statué en ce sens. Toutefois, bien que le respect de ces règlements soit ici un enjeu essentiel, puisqu'ils visent à atténuer les sentiments de vulnérabilité et d'humiliation des femmes dans pareilles circonstances, on aurait tort de centrer le débat uniquement sur de telles considérations. Car la principale question que cette intervention soulève est, avant tout, celle de sa légitimité même. Et à plus forte raison lorsqu'on prend connaissance de certains commentaires qui laissent entendre que les véritables motifs de l'intervention étaient d'apaiser les surveillants qui avaient manifesté à l'extérieur des murs de la prison, suite aux événements de la fin d'avril 1994.

Alors, à la suggestion de Monsieur Cayouette que “... ces vidéos risquent de court-circuiter le réflexe critique du téléspectateur ...," je rétorquerais que ce vidéo nous permet enfin de voir vraiment ce qui s'est passé et surtout de donner une crédibilité qu'on refusait initialement aux propos des femmes, puisque ces images remettent en question sérieusement les rapports internes. Ce qui m'inquiète ici est que malgré les témoignages des représentantes de la Association Canadienne des Sociétés Elizabeth Fry qui critiquaient cette intervention, il nous faudra attendre des images pour y croire.

\section{Le corps maté, "le corps docile"}

Les images sont troublantes. Les scènes ressemblent à des images pornographiques. Il y a aussi érotisation de la violence. La soumission des femmes mises à nu. Des gardiens, en l'occurrence des hommes, mâtant des femmes. Mâtant le corps des femmes. Pour les transformer en "corps docile." Une succession d'images de femmes nues, enchaînées et ne résistant pas. Un collègue me demandait s'il serait approprié de montrer ce vidéo à nos étudiants. Il était inconfortable. Il disait que de montrer des femmes nues ... c'était délicat. Bizarre, étant donné que l'on 
ne se gêne pas de montrer à nos étudiants des videos comme The Accused, Ce n'est pas une histoire d'amour, Vol au dessus d'un nid de coucou, I'Orange Mécanique et bien d'autres. Pourquoi, cette pudeur soudaine?

Une série de films existent sur les femmes en prison ${ }^{6}$ et servent très souvent de première impression de ce que sont les femmes détenues. Ces films hollywoodiens, en majorité, jouent sur des mythes et présentent les femmes en détention comme violentes, masculines, lesbiennes et ne remettent jamais en question les conditions de détention. L'idée véhiculée est que le problème réside uniquement chez les femmes et non aussi dans la nature de l'institution. Ce que changera ce vidéo. De plus, la réalisation de différents documentaires par l'Office national du film, depuis le début des années 1980, tente de présenter la voix des femmes, leurs expériences et leurs conditions de détention, comme dans $P 4 W$ (pénitencier de Kingston), C'est pas parce qu'on vit dans un château qu'on est les princesses (la Maison Gomin à Québec), Les bleus au coeur (la Maison Tanguay à Montréal ${ }^{7}$ ), To Heal the Spirit et Getting Out (sur les femmes autochtones à $\mathrm{P} 4 \mathrm{~W}$ ) et, tout récemment, A double tour (le pénitencier pour femmes francophones à Kingston). Donc, on tente d'utiliser le médium très puissant qu'est l'écran pour présenter une contrepartie $^{8}$. Réalité, fiction ou voyeurisme? A mon avis, comme outil pédagogique, ces documentaires ont une valeur pour susciter une réflexion et une discussion autour de la problématique des femmes en prison, l'intervention et la situation des femmes autochtones, notamment. Les images parlent aux étudiant-es parfois beaucoup plus que ne parlent les livres et/ou les articles théoriques.

\section{Sur l'enfermement carcéral dcs emmes}

Pour mieux situer la problématique de l'enfermement carcéral des femmes, il faut savoir que les femmes en prison représentent $7 \%$ de la population carcérale provinciale (sentencées à moins de deux ans) et $4 \%$ de la population carcérale fédérale (sentencées à plus de deux ans). Au Canada, en juin 1990, 305 femmes purgeaient des peines fédérales contre 13,234 hommes 9 . P4W est le seul pénitencier pour femmes au Canada, comparativement à plus de 40 établissements pour hommes. On y compte environ 150 femmes (entre $20 \%$ et $40 \%$ sont francophones) et quelques 150 autres sont incarcérées dans des institutions provinciales 
suite à un accord d'échange avec certaines provinces ${ }^{10}$, comme avec le Québec depuis 73, avec la Maison Tanguay, par exemple. Au total, on peut compter environ 800 lemmes dans les institutions provinciales canadiennes. Donc, s'il y a un seul pénitencier pour femmes au Canada, cela pose un certain nombre de difficultés. Une première se situe au niveau de la classification étant donné que toutes les femmes sont soumises au même type de contrôle même si les besoins de sécurité sont différents, contrairement à la situation qui prévaut dans les institutions masculines. Loin de moi de suggérer que les pénitenciers pour hommes sont des modèles à suivre, mais en ayant des "pens" à sécurité maximum, médium et minimum, on peut penser que l'on y offre un controle plus adapté aux besoins. Évidemment, I'incarcération est toujours un mécanisme de punition, de contrôle et de répression ${ }^{11}$.

Une autre difficulté à souligner par rapport à $\mathrm{P} 4 \mathrm{~W}$ est que les femmes provenant de toutes les provinces canadiennes sont incarcérées à Kingston, engendrant ainsi de sérieuses difficultés pour celles ayant des enfants, notamment. Ce qui est le cas de la majorité des femmes en détention. Or, contrairement aux hommes, elles n'ont généralement pas de conjoint à l'extérieur qui s'occupe des enfants.

Le nombre non négligeable de suicides entre les années 1977 et 1991 à $\mathrm{P} 4 \mathrm{~W}$ a aussi sonné l'alarme. Selon des sources non officielles, une douzaine de femmes, dont huit autochtones (elles représentent environ $25 \%$ des détenues au fédéral et sont donc largement sur représentées), se seraient enlevées la vie à l'intérieur des murs ${ }^{12}$. Cette situation d'automutilation a aussi mis en lumière le fait que les femmes incarcérées ont été très souvent dans le passé victimes d'inceste, d'abus physiques et psychologiques et de violence conjugale. Nous n'avons qu'à penser ici aux femmes qui tuent leur conjoint violent. Selon le rapport d'un groupe d'étude sur les femmes sentencées au fédéral, La Création de choix, plus de $80 \%$ des femmes incarcérées auraient été victimisées avant l'incarcération. Donc la violence qu'elles ont subie aux mains, la plupart du temps, d'hommes (E..: pères, frères, beaux-pères et conjoints) ne devrait pas être négligée lorsque l'on parle d'intervention en général et aussi d'intervention "musclée" et "masculine" auprès des femmes.

En 1990, La Création de choix recommandait la lermeture de Kingston pour l'année 1994 (repoussée maintenant à 1996) et l'ouverture de six centres régionaux de détention au Canada ${ }^{13}$. La fermeture de cette institution a été préconisée depuis presque son ouverture en 1934. 
Plusieurs commissions d'enquête se sont succédées pour toutes arriver à la même conclusion: la fermeture de $\mathrm{P} 4 \mathrm{~W}^{14}$. Plus de soixante ans ont passé.

\section{De l'indignation au silence}

Le public, les médias, les organismes s'occupant des droits des détenues, particulièrement par le biais de la directrice nationale de la Association Canadienne des Sociétés Elizabeth Fry, Madame Kim Pate, tous ont tour à tour dénoncé le caractère violent et non justifié de telles stratégies d'intervention. Quelques émissions se sont penchées plus sérieusement sur la question de la détention, comme à l'émission Le Point, mais sans porter une attention particulière aux femmes. L'émission de radio des dames Payette a, par ailleurs, posé des questions plus fondamentales quant à la situation des femmes détenues. Mais, après l'indignation spontanée, le silence. Et comme le soulignait en entrevue Madame Nathalie Duhamel de la Maison de transition Thérèse-Casgrain de Montréal, I'attention donnée à cet événement fut très brève et elle se demande s'il faut qu'une femme se tue pour que quelque chose soit fait; et encore.

Mais enfin, si vous vous souvenez bien, nous sommes passés aux choses sérieuses: la rentrée de Monsieur Lucien Bouchard, la visite de Clinton et la sortie du budget Martin. Pour ce qui est du rétablissement de la peine de mort dans l'État de New York ... passons. Silence, caméra, on tourne.

\section{ENDNOTES}

1 Une version abrégée de ce commentaire, portant le même titre, est parue dans l'édition du 21 mars 1995 du journal Le Devoir. Je tiens à remercier mon collègue, Fernando Acosta, pour ses précieux conseils sur cette première version.

2 La violence de ces images a rejoint beaucoup de femmes. Elles se sont aussi senties victimisées.

3 Il faut noter ici que lors d'une émission de Oprah au début des années 1990, un vidéo clandestin "mettant en vedette" la violence de gardiens sur des détenues aurait aussi fait scandale. Sur ce sujet, Karlene Faith dans Unruly Women: The Politics of Confinement and Resistance, Vancouver: Press Gang Publishers, 1993 à la page 249 note les faits suivants, qui, soit dit en passant, ressemblent étrangement au images de Kingston à l'exception peut-etre de la résistance de ces femmes: 
The program (NBC, March 31, 1993), opened with a clandestine videotape produced by a guard at the Georgia Women's Correctional Institution Main Unit (with a population exceeding 900 women). We see male and female guards struggle with resistant women, strip them naked, place them in restraining jackets, and leave them hogtied ankles and wrists bound at their backs) and chained in dark isolation cells.

4 Le Globe and Mail du mardi 21 février rapportait qu'il y avait une femme dans l'équipe d'intervention.

5 Voir Michel Foucault, Surveiller et Punir: Naissance de la Prison (Paris: Éditions Gallimard, 1975) pour une des meilleures discussions et analyses des "dispositifs" et mécanismes mis en place pour assurer l'assujettissement des corps et la transformation des corps récalcitrants en "corps dociles" dans le régime pénitentiaire. Pour une discussion du corps, à la fois comme site de contrôle et comme site de résistance, voir Journal of Prisoners on Prisons, vol. 5, no. 2, été 1994 et plus particulièrement un poème de Norma Stafford, "My blood", les sculptures de Gayle K. Horii, les témoignages de femmes autochtones, les expériences des femmes ayant le sida, I'automutilation et le suicide.

6 Notons, par exemple, Snake Pit (1948), Caged (1950) et Turning to Stone (1986). Pour une discussion plus en détails, voir à ce sujet Karlene Faith, le chapitre "Going to the Movies" dans supra note 3.

7 Notez l'utilisation du terme "maison" au lieu de "prison."

8 Par exemple, en septembre 1993, se tenait à Londres, un colloque intitulé Captured on Film: International Film Festival on Women and Imprisonment, présentant des films de plusieurs pays sur les femmes en prison. Le Canada y était représente par To Heal the Spirit et Getting Out.

9 Voir Statistiques Canada, 1990 à la page 1.

10 Voir Faith, supra note 3 à la page 138.

11 Dans ce sens, je me réjouis de lidée présentée par le ministre de la Justice du Québec, Monsieur Ménard, qui recommande la fermeture de certaines prisons québécoises dans le but de favoriser d'autres formes d'intervention. Il serait plutôt ironique que les difficultés budgétaires de l'État québécois contribuent à humaniser le traitement judiciaire des délinquants.

12 Voir Faith, supra note 3 à la page 139.

13 La construction de ces centres est prévue à Maple Creek en Saskatchewan pour les femmes autochtones ("Healing Lodge"); un autre à Edmonton en Alberta; un à Joliette au Québec; un à Kitchener en Ontario; et un à Truro en Nouvelle-Ecosse. Un centre pour femmes en Colombie-Britannique est déjà en opération depuis 1991. Voir: Services Correctionnels du Canada, La Création de Choix: Rapport du groupe d'étude sur les femmes purgeant une peine fédérale (Ministère des Approvisionnements et Services, avril 1990).

14 Dès 1938, le rapport Archambault recommandait la fermeture de P4W. 


\section{REFERENCES}

Commission Canadienne sur la détermination de la peine. (1938). Réformer la Sentence: Une approache Canadienne: Rapport de la Commission Canadienne sur la détermination de la peine.

Faith, Karlene. (1993). Unruly Women: The Politics of Confinement and Resentment. Vancouver: Press Gang Publishers.

Foucault, Michel. (1975). Surveiller et Punir: Naissance de la Prison. Paris: Éditions Gallimard.

Horii, Gayle K (1994). "Sculptures," Journal of Prisoners on Prisons, Volume 5:2. Services Correctionnels du Canada. (1990). La Crèation de Choix: Rapport du groupe d'étude sur les femmes purgeant une peine fédérale. Ministère des Approvisionnements et Services.

Stafford, Norma (1994). "My Blood," Journal of Prisoners on Prisons, Volume 5:2. Statistiques Canada, 1990, p. 1.

\section{FILMS}

To Heal the Spirit. (1990). Producer: Pen Glesser. Director: Barbara Barle. Produced by Why Not Productions, Inc. in association with the CBC. Published: Toronto: CBC Enterprises.

Getting Out. (1993). Producer-Director: Anne Pick. Produced by Why Not Productions, Inc. Published: Rexdale, Ontario: Mobius Media Corporation. 